\title{
GLAD4U: deriving and prioritizing gene lists from PubMed literature
}

\author{
Jérôme Jourquin ${ }^{1,2}$, Dexter Duncan ${ }^{1}$, Zhiao Shi ${ }^{3,4}$, Bing Zhang ${ }^{1,2^{*}}$ \\ From The International Conference on Intelligent Biology and Medicine (ICIBM) \\ Nashville, TN, USA. 22-24 April 2012
}

\begin{abstract}
Background: Answering questions such as "Which genes are related to breast cancer?" usually requires retrieving relevant publications through the PubMed search engine, reading these publications, and creating gene lists. This process is not only time-consuming, but also prone to errors.

Results: We report GLAD4U (Gene List Automatically Derived For You), a new, free web-based gene retrieval and prioritization tool. GLAD4U takes advantage of existing resources of the NCBI to ensure computational efficiency. The quality of gene lists created by GLAD4U for three Gene Ontology (GO) terms and three disease terms was assessed using corresponding "gold standard" lists curated in public databases. For all queries, GLAD4U gene lists showed very high recall but low precision, leading to low F-measure. As a comparison, EBIMed's recall was consistently lower than GLAD4U, but its precision was higher. To present the most relevant genes at the top of a list, we studied two prioritization methods based on publication count and the hypergeometric test, and compared the ranked lists and those generated by EBIMed to the gold standards. Both GLAD4U methods outperformed EBIMed for all queries based on a variety of quality metrics. Moreover, the hypergeometric method allowed for a better performance by thresholding genes with low scores. In addition, manual examination suggests that many false-positives could be explained by the incompleteness of the gold standards. The GLAD4U user interface accepts any valid queries for PubMed, and its output page displays the ranked gene list and information associated with each gene, chronologically-ordered supporting publications, along with a summary of the run and links for file export and functional enrichment and protein interaction network analysis.
\end{abstract}

Conclusions: GLAD4U has a high overall recall. Although precision is generally low, the prioritization methods successfully rank truly relevant genes at the top of the lists to facilitate efficient browsing. GLAD4U is simple to use, and its interface can be found at: http://bioinfo.vanderbilt.edu/glad4u.

\section{Background}

The physical development and phenotype of organisms can be thought of as a product of genes interacting with each other and with the environment. Therefore, it is common for a scientist to ask questions like "Which genes are related to breast cancer?", "Which genes are involved in embryonic development?", and "Which genes are functionally related to TP53?"

\footnotetext{
* Correspondence: bing.zhang@vanderbilt.edu

'Department of Biomedical Informatics, Vanderbilt University School of Medicine, 400 Eskind Biomedical Library, 2209 Garland Avenue, Nashville, TN 37232, USA

Full list of author information is available at the end of the article
}

The current answers to these questions are primarily contained in the articles indexed in the MEDLINE database. Traditionally, answering these questions requires individuals to retrieve relevant publications through the PubMed search engine and then to create gene lists by manually extracting gene-centered information from retrieved literature. This process is not only time-consuming, but also prone to errors. First, it is difficult to ascertain that all relevant literature is processed. Second, it is unlikely that all relationships in a publication will be detected. Third, individual researchers tend to extrapolate based on domain knowledge.

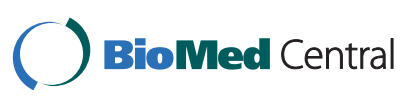


Over the past decade, bioinformatics approaches have been developed to address this issue. One of the most successful projects in this area is the Gene Ontology (GO) project [1]. GO produces a structured, precisely defined, and controlled vocabulary (i.e., GO terms) for describing the roles of genes and gene products in different species. Genes are associated with GO terms through manual curation as well as computational inference. A researcher can now go to the GO website [2] to get a list of genes related to a GO term of interest. However, as the GO vocabulary only describes gene products in terms of their associated biological processes, cellular components and molecular functions, users are limited by questions linked to this limited vocabulary. Moreover, processes, functions or components that are unique to diseases, such as oncogenesis, are not included in GO because causing cancer is not the normal function of any gene.

A useful resource specifically designed for disease studies is the Online Mendelian Inheritance in Man (OMIM [3]) project. OMIM is a comprehensive, authoritative, and timely compendium of human genes and genetic phenotypes. It contains information on all known Mendelian disorders. However, information on complex diseases such as cancer and diabetes is lacking in OMIM.

In addition to manual curation, text mining tools have been developed to assist gene list creation [4]. As an example, EBIMed [5,6] combines text mining with cooccurrence-based analysis to generate a prioritized list of genes for a user-provided query. Specifically, EBIMed collects MEDLINE records and available full text documents for a user-provided query, identifies protein names, drugs, species, or GO terms in the documents, and prioritizes genes/proteins based on the number of co-occurrences of the different pairs (protein/protein, protein/drug, protein/species, protein/GO term) in the sentences of the documents in which they appear. EBIMed and similar tools, such as FACTA [7] and SciMiner [8], provide more flexible ways to create gene lists that are not limited to certain aspects of biology. Nevertheless, they usually require heavy computation, and the relevance of the resulted gene lists to the input queries has not been systematically evaluated.

Here, we report GLAD4U (Gene List Automatically Derived For You), a new web-based gene retrieval and prioritization tool. GLAD4U takes advantage of existing resources at the National Center for Biotechnology Information (NCBI) to ensure computational efficiency. It provides a simple user interface that facilitates intuitive usage and interpretation of results. The quality of gene lists created by GLAD4U is assessed using corresponding "gold standard" lists curated in GO, GAD (Genetic Association Database [9]), and OMIM. The performance of GLAD4U is also compared with EBIMed.

\section{Results}

\section{Overall quality of the retrieved gene lists}

GLAD4U relies on the NCBI eSearch API to find publications related to a user query and on the gene-to-publication link table to identify genes from the retrieved publications. We used three GO biological process terms (apoptosis, cell adhesion and DNA repair) and three disease terms (hypertension, obesity and schizophrenia) as queries to evaluate the overall quality of the retrieved gene lists. For each query, using a corresponding gene list curated by GO or GAD/OMIM as a gold standard, we calculated the precision, recall and F-measure of the retrieved gene list. As shown in Table 1, gene lists retrieved for all queries showed very high recall $(0.90$ \pm 0.03 for $\mathrm{GO}$ terms and $0.96 \pm 0.05$ for disease terms). In contrast to the high recall, the precision was generally low $(0.16 \pm 0.04$ for GO terms and $0.06 \pm 0.02$ for disease terms), leading to low F-measures $(0.27 \pm 0.05$ for $\mathrm{GO}$ terms and $0.12 \pm 0.03$ for disease terms). EBIMed's recall is consistently lower than GLAD4U $(0.47 \pm 0.15$ for GO terms and $0.44 \pm 0.11$ for disease terms). However, its precision is higher than GLAD4U $(0.20 \pm 0.05$ for GO terms and $0.16 \pm 0.04$ for disease terms), resulting in better F-measures $(0.27 \pm 0.03$ for $\mathrm{GO}$ terms and $0.23 \pm 0.04$ for disease terms).

The low precision of GLAD4U may be partially attributed to the incompleteness of the annotation in $\mathrm{GO}$ and GAD/OMIM. However, it is likely that the original gene lists include many irrelevant genes. In this case, a prioritization step that ranks truly relevant genes at the top of a list would certainly facilitate efficient browsing.

\section{Performance of the prioritization methods}

We studied the performance of two methods to prioritize the gene lists. The first, "GLAD4U Counts", is based solely on the number of supporting publications as commonly implemented in other software $[10,11]$. The second, "GLAD4U Hypergeometric", is proposed in this study, which is based on the Hypergeometric test (see the Methods section for details). We used the above mentioned three GO terms and three disease terms as queries to evaluate the performance of our prioritization methods. We also included the prioritized gene lists returned by EBIMed for comparison.

Figure 1 depicts the precision/recall curves from this comparative evaluation. For all queries, based on manual inspection of the curves, both GLAD4U Counts and GLAD4U Hypergeometric outperformed EBIMed, especially at the high precision range. Between the two GLAD4U methods, the Hypergeometric method performed better than the Counts method for GO term queries, while their performances were comparable for disease term queries. The superior overall performance 
Table 1 Overall quality of the retrieved gene lists

\begin{tabular}{|c|c|c|c|c|c|c|}
\hline Query & GO/ MIM gene count & GLAD4U gene count & EBIMed gene count & & GLAD4U & EBIMed \\
\hline \multirow[t]{3}{*}{ Apoptosis } & 1039 & 6037 (958) & $1469(387)$ & Precision & 0.1587 & 0.2634 \\
\hline & & [195715] & [10000] & Recall & 0.9220 & 0.3725 \\
\hline & & & & F-measure & 0.2708 & 0.3086 \\
\hline \multirow[t]{3}{*}{ Cell adhesion } & 785 & $4195(691)$ & $1725(305)$ & Precision & 0.1647 & 0.1769 \\
\hline & & {$[125144]$} & {$[10000]$} & Recall & 0.8802 & 0.3885 \\
\hline & & & & F-measure & 0.2775 & 0.2431 \\
\hline \multirow[t]{3}{*}{ DNA repair } & 282 & $2476(263)$ & $1100(180)$ & Precision & 0.1062 & 0.1636 \\
\hline & & [60952] & [10000] & Recall & 0.9326 & 0.6383 \\
\hline & & & & F-measure & 0.1907 & 0.2605 \\
\hline \multirow[t]{3}{*}{ Hypertension } & 87 & $2046(77)$ & $135(27)$ & Precision & 0.0376 & 0.2000 \\
\hline & & [323818] & [10000] & Recall & 0.8851 & 0.3103 \\
\hline & & & & F-measure & 0.0721 & 0.2432 \\
\hline \multirow[t]{3}{*}{ Obesity } & 111 & $1778(110)$ & $350(59)$ & Precision & 0.0619 & 0.1686 \\
\hline & & [141615] & [10000] & Recall & 0.9910 & 0.5315 \\
\hline & & & & F-measure & 0.1165 & 0.2560 \\
\hline \multirow[t]{3}{*}{ Schizophrenia } & 94 & $1725(90)$ & $382(44)$ & Precision & 0.0522 & 0.1152 \\
\hline & & [91194] & [10000] & Recall & 0.9574 & 0.4681 \\
\hline & & & & F-measure & 0.0990 & 0.1849 \\
\hline
\end{tabular}

Numbers in parentheses indicate the number of genes overlapping between the GLAD4U or EBIMed lists and the corresponding gold standard, numbers in square brackets indicate the number of publications retrieved by the query.

of the two GLAD4U methods over EBIMed was further evaluated by computing AP, a quantitative measure of quality across all recall levels (Table 2). In this analysis, GLAD4U Counts and Hypergeometric methods scored better than EBIMed $(0.48 \pm 0.10,0.52 \pm 0.12$ and $0.21 \pm 0.09$, respectively), with GLAD4U Hypergeometric performing the best (Table 2).

The precision-recall curve and the AP score factor in precision at all recall levels. For ranked gene lists, particularly in web-based applications, this may not be of interest to users. In most scenarios, what matters may be the number of relevant genes on the first page or the first several pages. "Precision at $k^{\text {" }}$ is usually used to measure precision at a fixed low level of retrieved results, e.g., the top $k$ results. To this end, we calculated the precisions for the top $50(k=50)$ and top $100(k=$ $100)$ genes for all three methods, for each query (Table 2). GLAD4U Counts and GLAD4U Hypergeometric methods maintained higher precisions for the top 50 genes compared to EBIMed $(0.74 \pm 0.15,0.77 \pm 0.20$ and $0.54 \pm 0.18$, respectively), as well as for the top 100 genes $(0.64 \pm 0.20,0.69 \pm 0.25$ and $0.42 \pm 0.20$, respectively). Although the AP-based comparison may be biased against EBIMed owing to its low overall recall, precision at 50 and 100 only focus on the top ranking genes and are not affected by the overall recall. These results suggest that GLAD4U can produce lists where relevant genes are ranked at the top.
Although precision was less than perfect even for the top ranking genes, we noticed that many false-positives could be explained by the incompleteness of the gold standards. Table 3 lists the first 10 genes-along with their first 10 supporting publications-returned by GLAD4U Hypergeometric method that were not in the corresponding gold standards for the terms "apoptosis" and "hypertension" (see additional files 1 and 2 for the complete lists of genes and supporting publications). Taking the first and last genes in the list as examples, for each term (i.e., MDM2 and ING1 for apoptosis, and REN and ACE2 for hypertension), we found strong evidence in the most recent supporting publications for linking these non-gold standard genes to the query. MDM2 has antiapoptotic effects, and its direct interaction and regulation of p53 define it as an oncogene [12-15]. It translocates to the nucleus to interact with p53 and p300, promotes cell growth by initiating p53 degradation [16,17]. Its expression is directly linked to prostate cancer patient susceptibility [18]. Inhibitor of growth family, member 1 (ING1) is involved in cell stress and DNA damage response [19-22]. Up-regulation of p33ING1b or p24ING1c, two of the three alternatively spliced transcripts of ING1 resulted in increased early apoptotic cells $[23,24]$, probably through interactions with mdm2, p14arf, and lamin A $[25,26]$. This effect is dependent on the presence of functional p53 $[25,27]$ and the H3K3me3 binding domain of IGN1 [28]. 

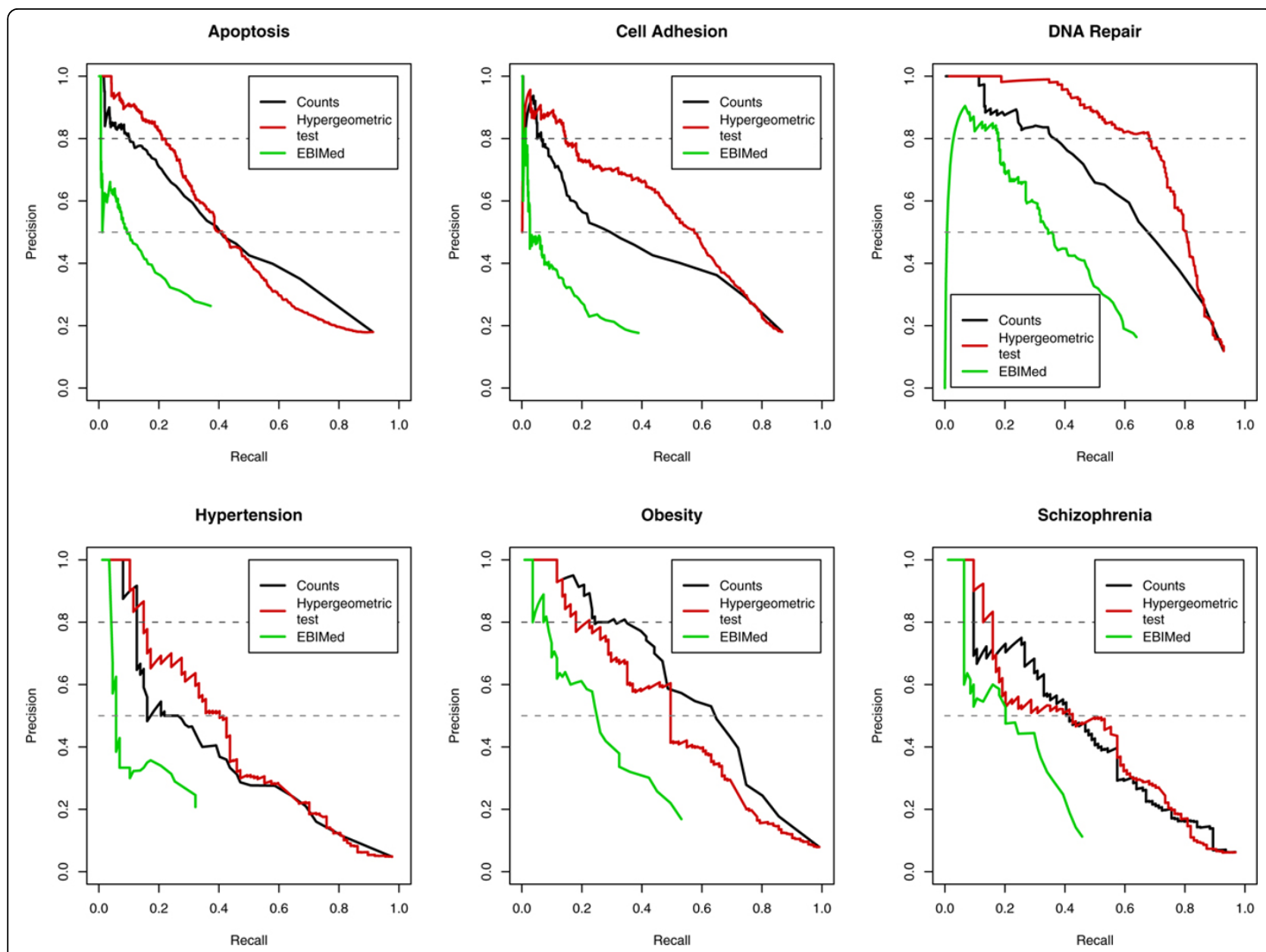

Figure 1 Precision/recall curves for different prioritization methods. Precision/recall curves for GLAD4U Counts, GLAD4U Hypergeometric and EBIMed are colored in black, red, and green, respectively. Dashed lines correspond to the precision levels of 0.8 and 0.5 .

Regarding hypertension, renin (REN) is part of the renin-angiotensin system (RAS). Proteins in this system are thought as important regulators of blood pressure and are involved in the onset of hypertension [29-32].

Table 2 Comparison of different prioritization methods

\begin{tabular}{|c|c|c|c|c|c|c|}
\hline & Apoptosis & Cell Adhesion & DNA Repair & Hypertension & Obesity & Schizophrenia \\
\hline \multicolumn{7}{|l|}{ GLAD4U Counts } \\
\hline AP & 0.4939 & 0.4611 & 0.6670 & 0.3947 & 0.5698 & 0.4601 \\
\hline Precision at $k=50$ & 0.8600 & 0.8000 & 0.8800 & 0.4800 & 0.7800 & 0.5400 \\
\hline Precision at $k=100$ & 0.8300 & 0.7300 & 0.8100 & 0.3800 & 0.5500 & 0.4200 \\
\hline \multicolumn{7}{|c|}{ GLAD4U Hypergeometric } \\
\hline AP & 0.4942 & 0.5723 & 0.8139 & 0.4564 & 0.4782 & 0.4280 \\
\hline Precision at $k=50$ & 0.9400 & 0.9000 & 1.0000 & 0.5800 & 0.6200 & 0.4800 \\
\hline Precision at $k=100$ & 0.9000 & 0.8500 & 0.9700 & 0.3900 & 0.5200 & 0.4400 \\
\hline \multicolumn{7}{|l|}{ EBIMed } \\
\hline$\overline{\mathrm{AP}}$ & 0.1567 & 0.1256 & 0.3517 & 0.1336 & 0.2673 & 0.2318 \\
\hline Precision at $k=50$ & 0.6200 & 0.4800 & 0.8400 & 0.3137 & 0.5652 & 0.4423 \\
\hline Precision at $k=100$ & 0.5980 & 0.4848 & 0.6700 & 0.2821 & 0.1586 & 0.3200 \\
\hline
\end{tabular}

AP: Average Precision 
Table 3 First 10 genes retrieved by GLAD4U and not listed in the gold standard lists

\begin{tabular}{|c|c|c|c|}
\hline Rank & $\begin{array}{l}\text { Entrez-Gene ID } \\
\text { (Gene symbol) }\end{array}$ & Score & PMIDs* \\
\hline \multicolumn{4}{|c|}{ Apoptosis } \\
\hline 41 & 4193 (MDM2) & 53.5212 & $21051655,21051533,20849854,20849851,20832750,20822933,20708156,20659896,20657550,20644561$ \\
\hline 48 & 1432 (MAPK14) & 40.8288 & 20736797, 20573801, 20558744, 20473571, 20463961, 20430109, 20393480, 20345980, 20307495, 20299663 \\
\hline 49 & 4609 (MYC) & 37.27 .98 & $20714214,20598117,20596624,20573831,20564213,20515470,20232342,20071475,19996270,19966300$ \\
\hline 54 & 6774 (STAT3) & 35.2695 & $20562100,20514402,20507639,20490331,20459702,20447714,20213502,20197401,20164027,20154216$ \\
\hline 77 & 5580 (PRKCD) & 23.3017 & 20548952, 20547768, 20471435, 20093486, 19932628, 19917613, 19875824, 19833733, 19808702, 19747914 \\
\hline 78 & $29126(C D 274)$ & 23.1218 & 20636820, 20617899, 20587542, 20506224, 20445553, 20363965, 19916867, 19826049, 19811426, 19794071 \\
\hline 79 & 142 (PARP1) & 22.9308 & $20940411,20665026,20644561,20629644,20564216,20453000,20388712,20181890,20177052,20072652$ \\
\hline 86 & 406991 (MIR21) & 18.9856 & 20813833, 20515755, 20514462, 20447717, 20404348, 20372781, 20371612, 20346171, 20153722, 20148895 \\
\hline 96 & $7295($ TXN) & 16.1886 & 20619274, 20430109, 20298786, 20103619, 19671194, 19566940, 19328186, 19120277, 18983687, 18848838 \\
\hline 100 & $3621($ ING1) & 15.3784 & $19085961,18836436,18801192,18691180,18655775,18533182,18388957,17585055,17379210,16607280$ \\
\hline \multicolumn{4}{|c|}{ Hypertension } \\
\hline 10 & 5972 (REN) & 61.9237 & 20925572, 20662730, 20577119, 20537141, 20429690, 20223792, 20160196, 19891555, 19673942, 19536175 \\
\hline 12 & 3291 (HSD11B2) & 45.7032 & $20597806,19811365,19150652,18837962,18573267,18178212,17551100,16872738,16778331,16109323$ \\
\hline 14 & 4879 (NPPB) & 36.9570 & 20713912, 20368210, 20350538, 20346360, 20234137, 20142024, 20113292, 20102554, 20087954, 20083731 \\
\hline 17 & 4524 (MTHFR) & 32.2080 & $21072525,21060006,20960113,20852445,20812180,20717043,20669348,20637366,20592457,20479155$ \\
\hline 19 & $1401(\mathrm{CRP})$ & 31.9446 & 21044781, 20805569, 20733302, 20683147, 20676960, 20346360, 20339115, 20184533, 20074254, 20068351 \\
\hline 20 & 4878 (NPPA) & 31.6082 & 20577119, 20543198, 20368210, 20346360, 20137368, 19635983, 19479237, 19430483, 19346663, 19330901 \\
\hline 21 & 155 (ADRB3) & 28.4824 & $20831043,20144152,20044737,19842096,19779464,19479237,19131662,18724972,18510051,18088254$ \\
\hline 24 & 1584 (CYP11B1) & 24.8304 & 20708777, 20339375, 19820005, 19567537, 19082699, 18663314, 18294861, 17980006, 17296872, 17121536 \\
\hline 27 & 59272 (ACE2) & 21.4649 & $20831027,20813695,20679547,20349406,20160196,20117991,19926873,19684612,19289653,19286756$ \\
\hline 29 & 9370 (ADIPOQ) & 19.6898 & $21044781,20593932,20552610,20528971,20516205,20443850,20385503,20376890,20166815,20150538$ \\
\hline
\end{tabular}

Overexpression of REN leads to hypertension via chronic overproduction of AngII [33,34], and inhibiting the regulators of the RAS-such as REN-is a common treatment for hypertension [32]. Adiponectin (ADIPOQ) is an adipocytokine synthesized by the adipose tissue. It has been proposed as a biomarker for hypertension, as low plasma levels correlates with higher risk of hypertension [35-38], and possibly with coronary artery disease, kidney disease, left ventricular hypertrophy, and even myocardial infarction [36,39-41]. Interestingly, REN and ADIPOQ also present polymorphisms, which seem linked to therapeutic response to hypertension [31,40,42-46].

From these publications, we believe that MDM2 and IGN1 should be part of the apoptosis list, as well as REN and ADIPOQ should be part of the hypertension list. These results accentuate the incompleteness of the gold standards and suggest that GLAD4U can help in the completion of the gold standard lists.

\section{Thresholding score to enhance GLAD4U performance}

To evaluate whether thresholding the gene score can enhance GLAD4U performance, we acquired a broader list of disease-associated gene lists curated by Kohler et al.
[47] and available from the GeneWanderer website (http://compbio.charite.de/genewanderer). We extracted 32 "disease-gene families" to use as standards for evaluating GLAD4U performance before and after thresholding. On average, GLAD4U performs 2.90-time better when genes with low prioritization scores (i.e. prioritization score $<2$ or hypergeometric $p$ value $>0.01$ ) are removed, as illustrated by comparing the F-measures (Figure 2). The most increased performances were achieved for terms such as "prostate cancer", "obesity", and "amyotrophic lateral sclerosis" (folds of 7.28, 5.72, and 5.48, respectively) (see additional file 3 for the before and after F-measures, and corresponding fold-changes). The performances that least benefited from thresholding the gene list included "Noonan Syndrome, Costello syndrome, Cardiofaciocutaneous Syndrome", "Nonsyndromic hearing loss", and "Chondrodysplasia punctata" (folds of 1, 1.16, and 1.17 respectively).

\section{User interface}

GLAD4U uses a simple query interface for users to submit their queries. Any queries that are valid in a PubMed search can be used in GLAD4U. In the query interface, users can also modify the default parameters of the application, 


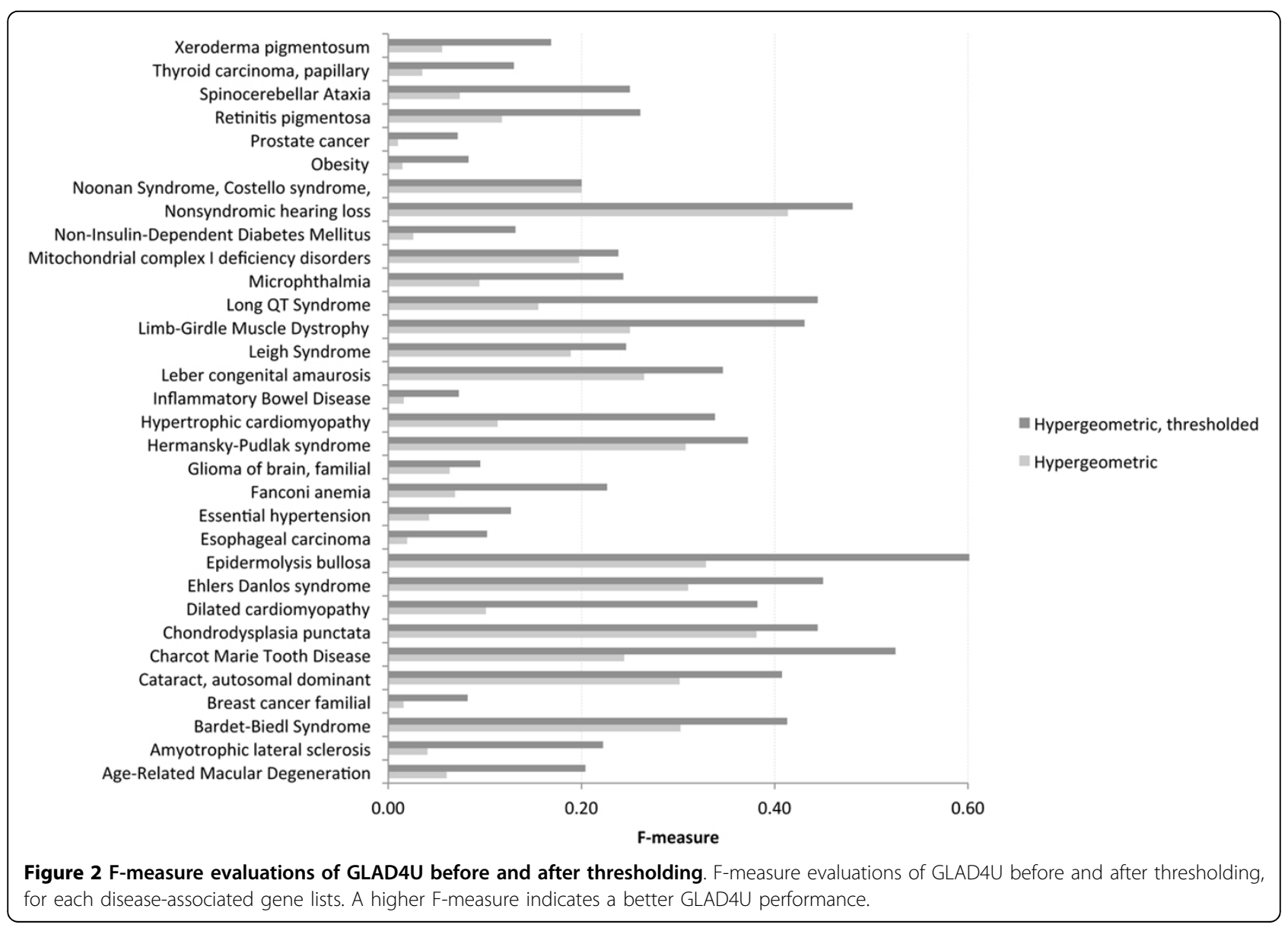

including: search space (all species or restricted to human genes), the number of genes to present per result page, the maximum number of publications supporting each gene returned in the result page and the number of pages to build for each of the algorithm runs.

The output page displays the ranked gene list and information associated with each gene (Figure 3). As each gene is identified by an Entrez-Gene ID, we use eSummary, another NCBI's eUtility [48], to fetch annotations for the gene including name, symbol and species. Publications supporting the relationship between a gene and the query term are listed under the gene. The publications are ordered based on their PubMed IDs so that the most recent publication is listed first (see Figure 3, under the "ADIPOQ" gene description). As for genes, we use eSummary to fetch information for the publication such as title, author and journal name. Genes and publications are hyperlinked to the corresponding NCBI pages, which will-by design-open in a new window to avoid disrupting the result page.

At the top of the output page, a summary of the run is also given: query term and options chosen, number of genes and publications processed, as well as a hyperlink to download the complete results in the comma-separated values (CSV) format. Although this file may be difficult to interpret by humans, it can be used as input for other computational analysis tools. For example, we have implemented a "send data to Functional Enrichment Analysis" link in the result page (Figure 3) of GLAD4U for submitting a gene list to the functional enrichment analysis tool WebGestalt $[49,50]$. This function is particularly handy for the functional interpretation of a gene list, e.g., a list returned by a disease term query. It could help revealing biological processes associated with the disease. As an example, enrichment analysis on the first 100 genes returned by the "Obesity" query linked this disease to biological processes such as "fat cell differentiation" (20 genes, multiple-test adjusted enrichment $\mathrm{p}$-value (adjp) = $5.27 \mathrm{e}-28)$, "lipid metabolic process" (39 genes, adjp = $5.05 \mathrm{e}-20)$ and "response to insulin stimulus" (17 genes, adjp $=4.99 \mathrm{e}-18$ ). In addition, we have also implemented a "visualize genes in a protein-protein interaction network" link, which allows the visualization of interactions among the protein products of the genes based on the Cytoscape Web utility (http://cytoscapeweb.cytoscape.org/). 


\section{GLAD4U - Vanderbilt University}

Department of Biomedical Informatics - $h$ ttp://bioinfo.vanderbilt.edu/glad $4 u$

Home | News/Updates | Documentation | Contact Us ${ }^{-1}$ Search 8

Ticket number: aue4duflq8u09com 9mbk34kj06

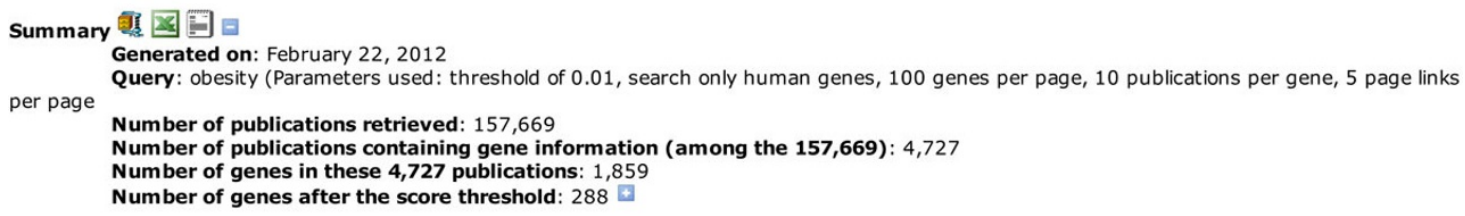

\section{Genes identified in your query, from highest to lowest scores:}

(all links will open in new windows)

Expand all publications

1. ADIPOQ - adiponectin, C1Q and collagen domain containing [Homo sapiens, Entrez-Gene ID:9370] score: 1000 , go to Entrez-Gene page, hide supporting publications

o Sitonen Net al., Association of ADIPOQ gene variants with body weight, type 2 diabetes and serumadiponectin concentrations: the Finnish Diabetes Prevention

- Sitonen N et al.,Association of ADIPOQ gene variants with body weight, type 2 diabetes and serum adiponectin concentrations: the Finnish Diabetes Prevention
Study. BMC Med Genet, 2011 Jan 10;12:5 - Abstract Sabouri S et al., Association be

- Thyagarajan B et al.,Serum adiponectin is positively associated with lung function in young adults, independent of obesity: the CARDIA study. Respir Res, 2010 Dec 9:11:176 - Abstract.

- Cohen SS et al.,Serumadiponectin in relation to body mass index and other correlates in black and white women. Ann Epidemiol, 2011 Feb;21(2):86-94 -

Abstract

- Choi KM et al.,Serum adipocyte fatty acid-binding protein, retinol-binding protein 4, and adiponectin concentrations in relation to the development of the metabolic syndrome in Korean boys: a 3-y prospective cohort study. Am J Clin Nutr, 2011 Jan;93(1): 19-26 - Abstract

- Sun $\mathrm{Het}$ al.,Adiponectin reduces C-reactive protein expression and downregulates STAT3 phosphorylation induced by IL-6 in HepG2 cells. Mol Cell Biochem. 2011 Jan:347(1-2):183-9 - Abstract

- Dimitri P et al.,Adipokines, bone-derived factors and bone turnover in obese children; evidence for altered fat-bone signalling resulting in reduced bone mass.

Bone, 2011 Feb;48(2):189-96 - Abstract

- Elias Eet al.,Erythrocyte sodium-lithium countertransport activity is inversely correlated to adiponectin, retinol binding protein 4 and body height. Scand J Clin Lab Invest, 2010 Nov;70(7):487-91 - Abstract

- Cieslak J et al..,Polymorphisms in 5'-flanking regions of genes encoding adiponectin, leptin, and resistin are not associated with obesity of Polish children and adolescents. Mol Biol Rep, 2011 Mar;38(3): 1793-8 - Abstract

$\circ$ Liu J et al., Adiponectin levels in non-obese first-degree relatives of type 2 diabetes patients and non-diabetic subjects: a 5-year follow-up study. J Int Med Res, 2010 May-Jun; 38(3):792-802 - Abstract

- see all supporting publications in PubMed

2. LEP - leptin [Homo sapiens, Entrez-Gene ID:3952]

score: 321.9260 , go to Entrez-Gene page, show the first 10 out of the 300 supporting publications 5

3. FTO - fat mass and obesity associated [Homo sapiens, Entrez-Gene ID:79068] score: 276.2372 , go to Entrez-Gene page, show the first 10 out of the 184 supporting publications 1

4. MC4R - melanocortin 4 receptor [Homo sapiens, Entrez-Gene ID:4160] score: 214.5474 , go to Entrez-Gene page, show the first 10 out of the 149 supporting publications

5. ADRB3 - adrenergic, beta-3-, receptor [Homo sapiens, Entrez-Gene ID:155] score: 145.6010 , go to Entrez-Gene page, show the first 10 out of the 122 supporting publications

6. LEPR - leptin receptor [Homo sapiens, Entrez-Gene ID:3953] score: 140.0002 , go to Entrez-Gene page, show the first 10 out of the 129 supporting publications

7. PPARG - peroxisome proliferator-activated receptor gamma [Homo sapiens, Entrez-Gene ID:5468] Score: 131.9575 , go to Entrez-Gene page, show the first 10 out of the 195 supporting publications

8. GHRL - ghrelin/obestatin prepropeptide [Homo sapiens, Entrez-Gene ID:51738] score: 114.4299 , go to Entrez-Gene page, show the first 10 out of the 129 supporting publications

9. RETN - resistin [Homo sapiens, Entrez-Gene ID:56729] score: 104.9504 , go to Entrez-Gene page, show the first 10 out of the 96 supporting publications

10. UCP2 - uncoupling protein 2 (mitochondrial, proton carrier) [Homo sapiens, Entrez-Gene ID:7351] score: 89.6734 , go to Entrez-Gene page, show the first 10 out of the 80 supporting publications

11. UCP3 - uncoupling protein 3 (mitochondrial, proton carrier) [Homo sapiens, Entrez-Gene ID:7352]

Figure 3 GLAD4U output page. A typical result page generated by a query with GLAD4U. The summary section presents the main statistics for the query, along with two hyperlinked icons to download the results as an entire archive of all pages of results ("compressed" icon), a CSV ("Excel" icon) or a text ("text" icon) file. Right below the summary, a link is available to send the results for functional enrichment analysis. In the main result section, the prioritized genes are presented. The user can click the " + " to show/hide the supporting publications, which are all hidden by default to help the read-out of the gene information. ADIPOQ gene is presented with its supporting publications as an example. 


\section{Discussion}

Reading through all relevant literature to generate a gene list is time consuming [10,51-53], a common concern that came up in all interviews of experimentalists that we performed (results not shown). GLAD4U addresses this problem by automatically creating a ranked list of genes following a user's input query.

One important feature of GLAD4U is its information processing. Based on our survey among experimentalists, GLAD4U follows the exact same steps that an experimentalist would follow: gather literature, extract gene information and create an expert list [54]. Whether a user queries a disease, a non-disease phenotype, a biological process or a gene, GLAD4U will fetch corresponding biomedical publications using NCBI's eUtilities API, retrieve relevant gene information, rank them and send them back to the user. GLAD4U ensures computational efficiency through effective use of existing NCBI resources, which also made it one of the winning applications in the National Library of Medicine (NLM)'s 2011 Software Development Challenge on the Innovative Uses of NLM Information.

Another important feature of GLAD4U is its simplicity. Researchers will be at ease using GLAD4U because its searching engine is powered by PubMed's API $[48,52]$, and behaves similarly to Entrez-PubMed [55]. GLAD4U outputs a clean result page where the user can easily find genes relevant to the concept queried and supporting publications. Additionally, the use of PubMed's API makes GLAD4U almost maintenance-free. GLAD4U will update itself along with the MEDLINE library update. This will ensure that GLAD4U's results will always be up-to-date with the current literature.

Several tools rely on PubMed to build disease candidate genes lists [5,8,52,56,57]. EBIMed [5] and FACTA [7] are concept-oriented applications for mining existing biomedical literature. They attempt to automatically establish the publication-concept (including genes) relationship through in-house text mining tools whereas GLAD4U relies on the manually curated publication-gene mapping provided by NCBI. According to our results, manual mapping seems to have notable impact on performance. Nevertheless, automated mapping would allow flexibility in extending the services for concepts other than genes.

Although using the biomedical literature as a knowledge source seems intuitive $[51,58,59]$, certain limitations exist: the literature is indexed based on titles, abstracts and keywords, not on full-text [60,61]. Thus, a set of publications retrieved may be incomplete (i.e., some publications relevant to the concept queried will not be retrieved because they do not contain the necessary keywords in their titles or abstracts) [62]. There is a possible bias in using the biomedical literature and ontology [55], as the most studied genes (those with the most publications) will have more weight $[51,63]$ at the expense of more relevant genes that might only be featured in few papers [64]. Thus, we use the hypergeometric test to rank genes based on how likely it would be to retrieve them by chance alone, based on the number of publications retrieved for this gene among the total number of publications linked to this gene. The less likely it is-the smaller the $p$ value-the higher the score will be for the gene. Thus, even if GLAD4U is solely retrieving its data from the biomedical literature, it prioritizes following a statistical analysis of the retrieved data.

The most obvious usage of GLAD4U is to generate a gene list for an input concept, which has been demonstrated in this paper. This can be extremely useful for the design of targeted high-throughput experiments. If one needs to create a custom array or selected proteins for targeted quantitative proteomic analysis using the selected reaction monitoring (SRM) assay, one can use GLAD4U and review the ranked list of genes that likely should be included in the experimental design. Besides generating gene lists for individual concepts, GLAD4U is very flexible and allows production of gene lists related to multiple concepts, which cannot be done by searching GO or OMIM databases. For example, a query of "smoking AND cancer" can generate a gene list that could potentially help exploring gene-environment interactions in cancer. GLAD4U also holds the potential to assist in improvement of the functional annotation of genes. Although GO contains more than 17,000 terms [4,65] and is regularly used in the bioinformatics field as a standard $[4,66]$, it is not complete $[51,67]$. Through manual checking of the top genes returned by GLAD4U that were not part of the gold standard lists, we easily found evidence that these genes were indeed linked to the query, and probably should have been included in the gold standard.

Finally, because GLAD4U prioritization algorithm assigns scores to genes, removing the genes with a low score consistantly improves the quality of the results. This result justifies thresholding GLAD4U results by default.

\section{Conclusions}

GLAD4U is a freely available web-application for creating expert candidate gene lists tailored to a user's query. It follows the same steps that the experimentalist would follow: gather literature, extract gene information and create an expert list. The simple interface of GLAD4U ensures easy usage and interpretation. Because GLAD4U relies on existing biomedical literature, it has an immediate credibility with experimentalists, who use this resource as a primary means for enhancing their knowledge and expertise. Although the gene list directly returned from a PubMed 
query is usually lengthy and noisy, the prioritization method implemented in GLAD4U successfully ranks truly relevant genes at the top of the list and facilitates efficient browsing of the list.

\section{Methods}

\section{Publication retrieval}

GLAD4U relies on the eSearch application programming interface (API) developed by the NCBI for retrieving publications from the MEDLINE database [48]. For a user query, eSearch returns an XML file containing the number of publications returned by the query and all publication identification IDs (PMIDs). The XML file is parsed to get the list of PMIDs associated with a user query.

\section{Gene retrieval}

Genes associated with PMIDs are retrieved based on the gene-to-publication link table provided by Entrez-Gene [68]. Links between Entrez-Gene IDs and PMIDs are created based on both manual curation within the NCBI and integration of information from other public databases. Publications linked to more than 500 genes are removed from the link table because they lack specificity. After this process, the link table included 3,509,732 genes and 647,523 publications for all organisms, among which 30,343 genes and 306,487 publications were related to human (as of 05/14/2011).

\section{Gene prioritization}

We studied two methods to prioritize the retrieved genes based on publication counts or the hypergeometric test. To prioritize using counts ("GLAD4U Counts"), each gene receives a score equal to the number of publications describing it in the link table. The other method ("GLAD4U Hypergeometric") uses the hypergeometric test to prioritize all retrieved genes. Specifically, for a given query $\mathrm{Q}$ and a gene $\mathrm{G}$, let $n$ be the number of publications retrieved for the query and present in the geneto-publication link table (query-relevant publications) and $k$ be the number of query-relevant publications that involves the gene G. Let us further assume that there are $m$ publications in the gene-to-publication link table, $j$ of which involve the gene $\mathrm{G}$ (gene-relevant publications). This method calculates the probability of observing $k$ or more query-relevant publications for the gene by chance, based on the hypergeometric test and scores the gene using the following formula:

$$
\begin{aligned}
& S_{G}=-\log _{10} f(m, n, j, k), \text { where } \\
& f_{(m, n, j, k)}=\sum_{i=k}^{\min (n, j)} \frac{\left(\begin{array}{c}
m-j \\
n-i
\end{array}\right)\left(\begin{array}{l}
j \\
i
\end{array}\right)}{\left(\begin{array}{c}
m \\
n
\end{array}\right)}
\end{aligned}
$$

\section{Performance evaluation}

We used GO and disease terms as queries to evaluate the performance of the GLAD4U algorithms. Gene lists curated in GO, OMIM and GAD [69] were used as a gold standard (i.e. relevant genes). We developed a Perl script to parse the files "gene2go.gz" [68] and "gene_ontology.1_2.obo" [70] in order to generate gene lists for GO terms (as of 12/20/2009). Because of the parent-child relationship among the GO terms as described in the GO Direct Acyclic Graph, genes with granular annotations were associated with their parent terms using the Perl script. Using GAD, we identified all genes associated to a disease term. Using OMIM, we retrieved all IDs prefixed with "\%" and "\#" with the query in the title. Corresponding gene IDs were mapped by parsing the file "mim2gene" [68] (as of 12/ $22 / 2009$ ). For each disease term, the lists obtained with GAD and OMIM were merged to serve as a gold standard. Retrieval performance was evaluated using precision, recall and F-measure. The F-measure is calculated by $2 p r /(p+r)$, where $p$ is the precision defined as $\mid\{$ relevant genes $\} \cap\{$ retrieved genes $\}|/|$ retrieved genes $\} \mid$ and $r$ is the recall defined as $\mid$ \{relevant genes $\} \cap\{$ retrieved genes $\}|/|\{$ relevant genes $\} \mid$. We used the precision/recall curve, average precision (AP) and precision at the top $k$ retrieved genes $(k=50$ and $k=100)$ to evaluate the performance of our gene prioritization methods, and compared it to the performance of the ranked lists generated by EBIMed [6]. All performance values are expressed in the text as mean \pm standard deviation.

\section{Web implementation}

The GLAD4U user interface was developed in HTML and PHP languages. The scripts to deploy and update the algorithm on web servers were written in Perl, while the generation of hypergeometric test scores is using C. JQuery was used to implement user-features such as the ability to hide/ show options and functions. An email notification module was implemented to allow users to retrieve their results at a later time. GLAD4U (http://bioinfo.vanderbilt. $\mathrm{edu} / \operatorname{glad} 4 \mathrm{u}$ ) is platform-independent and under a GNU GPL license [71]. It was tested on Internet Explorer 5.0, Firefox 3.0, Safari 3.0, Chrome, Netscape 7 or any higher versions of these browsers.

\section{Additional material}

Additional file 1: False-positive genes retrieved by querying "apoptosis" with GLAD4U. This table shows all genes retrieved by GLAD4U with the query "apoptosis" that were not among the gold standards. The table presents the rank and score of these genes and all the retrieved supporting publications.

Additional file 2: False-positive genes retrieved by querying

"hypertension" with GLAD4U. This table shows all genes retrieved by GLAD4U with the query "hypertension" that were not among the gold 
standards. The table presents the rank and score of these genes and all the retrieved supporting publications.

Additional file 3: GLAD4U prioritization of disease candidate genes. This table shows the number of genes associated with each GeneWanderer hereditaty disease, retrieved by GLAD4U and overlapping between the two lists before and after thresholding. F-measure fold change between the GLAD4U prioritized list before and after thresholding, as well as the actual F-measures are also displayed in the table.

\section{List of abbreviations used}

ADIPOQ: adiponectin; API: application programming interface; CSV: commaseparated values; GAD: genetic association database; GLAD4U Counts: GLAD4U prioritization algorithm using counts; GLAD4U Hypergeometric: GLAD4U prioritization algorithm using the hypergeometric test; GLAD4U: gene list automatically derived for you; GO: gene ontology; GOTM: GOTree Machine; ING1: inhibitor of growth family: member 1; AP: average precision NCBI: national center for biotechnology information; OMIM: online mendelian inheritance in man; PMIDs: publication identification IDs; REN: renin; SRM: selected reaction monitoring.

\section{Acknowledgements}

We thank Dr. Hua Xu for useful comments on the analysis and Ms. Brandy Weidow for proofreading the manuscript. We appreciate the users who provided useful information through our interviews and online survey. This work was supported by the National Institutes of Health $(\mathrm{NIH}) /$ National Institute of General Medical Sciences (NIGMS) through grant R01GM088822, the $\mathrm{NIH} /$ National Cancer Institute (NCl) through grant U54CA113007, and the $\mathrm{NIH}$ / National Institute of Mental Health (NIMH) through grant P50MH078028

This article has been published as part of BMC Genomics Volume 13 Supplement 8, 2012: Proceedings of The International Conference on Intelligent Biology and Medicine (ICIBM): Genomics. The full contents of the supplement are available online at http://www.biomedcentral.com/ bmcgenomics/supplements/13/S8.

\section{Author details}

'Department of Biomedical Informatics, Vanderbilt University School of Medicine, 400 Eskind Biomedical Library, 2209 Garland Avenue, Nashville, TN 37232, USA. '2Department of Cancer Biology, Vanderbilt University School of Medicine, 2220 Pierce Avenue, PRB771, Nashville, TN 37232, USA. ${ }^{3}$ Advanced Computing Center for Research \& Education, Vanderbilt University, Nashville, TN 37240, USA. ${ }^{4}$ Department of Electrical Engineering and Computer Science, Vanderbilt University, Nashville, TN 37240, USA

\section{Authors' contributions}

BZ and JJ conceived of the study, which was coordinated by BZ. JJ carried out the work with PHP and Perl, DD implemented the $C$ version of the algorithm, ZS implemented the cytoscape web plugin for network visualization. JJ, DD, ZS and BZ participated in testing. JJ and BZ participated in the analysis of the results and in writing of the manuscript.

\section{Competing interests}

The authors declare that they have no competing interests.

Published: 17 December 2012

\section{References}

1. Ashburner M, Ball CA, Blake JA, Botstein D, Butler H, Cherry JM, Davis AP, Dolinski K, Dwight SS, Eppig JT, et al: Gene ontology: tool for the unification of biology. The Gene Ontology Consortium. Nat Genet 2000, 25(1):25-29.

2. The Gene Ontology. [http://www.geneontology.org/].

3. Online Mendelian Inheritance in Man. [http://www.ncbi.nlm.nih.gov/ omim/]

4. Erhardt RA, Schneider R, Blaschke C: Status of text-mining techniques applied to biomedical text. Drug Discov Today 2006, 11(7-8):315-325.
5. Rebholz-Schuhmann D, Kirsch H, Arregui M, Gaudan S, Riethoven M, Stoehr P: EBIMed-text crunching to gather facts for proteins from Medline. Bioinformatics 2007, 23(2):e237-244.

6. EBIMed. [http://www.ebi.ac.uk/Rebholz-srv/ebimed/index.jsp].

7. Tsuruoka Y, Tsujii J, Ananiadou S: FACTA: a text search engine for finding associated biomedical concepts. Bioinformatics 2008, 24(21):2559-2560.

8. Hur J, Schuyler AD, States DJ, Feldman EL: SciMiner: web-based literature mining tool for target identification and functional enrichment analysis. Bioinformatics 2009, 25(6):838-840.

9. GAD. [http://geneticassociationdb.nih.gov/].

10. Becker KG, Hosack DA, Dennis G, Lempicki RA, Bright TJ, Cheadle C, Engel J: PubMatrix: a tool for multiplex literature mining. BMC Bioinformatics 2003, 4:61.

11. Tanabe L, Scherf U, Smith LH, Lee JK, Hunter L, Weinstein JN: MedMiner: an Internet text-mining tool for biomedical information, with application to gene expression profiling. Biotechniques 1999, 27(6):1210-1214, 12161217.

12. Castera L, Sabbagh A, Dehainault C, Michaux D, Mansuet-Lupo A, Patillon B, Lamar E, Aerts I, Lumbroso-Le Rouic L, Couturier J, et al: MDM2 as a modifier gene in retinoblastoma. J Natl Cancer Inst 2010, 102(23):1805-1808

13. Nardinocchi L, Puca R, Givol D, D'Orazi G: Counteracting MDM2-induced HIPK2 downregulation restores HIPK2/p53 apoptotic signaling in cancer cells. FEBS Lett 2010, 584(19):4253-4258.

14. Post SM, Quintas-Cardama A, Pant V, Iwakuma T, Hamir A, Jackson JG, Maccio DR, Bond GL, Johnson DG, Levine AJ, et al: A high-frequency regulatory polymorphism in the $\mathrm{p} 53$ pathway accelerates tumor development. Cancer Cell 2010, 18(3):220-230.

15. Yan J, Di Y, Shi H, Rao H, Huo K: Overexpression of SCYL1-BP1 stabilizes functional $\mathrm{p} 53$ by suppressing MDM2-mediated ubiquitination. FEBS Lett 2010, 584(20):4319-4324.

16. Phillips A, Teunisse A, Lam S, Lodder K, Darley M, Emaduddin M, Wolf A, Richter J, de Lange J, Verlaan-de Vries M, et al: HDMX-L is expressed from a functional p53-responsive promoter in the first intron of the HDMX gene and participates in an autoregulatory feedback loop to control p53 activity. J Biol Chem 2010, 285(38):29111-29127.

17. Lai KP, Leong WF, Chau JF, Jia D, Zeng L, Liu H, He L, Hao A, Zhang H, Meek $\mathrm{D}$, et al: $\mathrm{S} 6 \mathrm{~K} 1$ is a multifaceted regulator of $\mathrm{Mdm} 2$ that connects nutrient status and DNA damage response. EMBO J 2010, 29(17):2994-3006.

18. Mandal RK, Mittal RD: Are cell cycle and apoptosis genes associated with prostate cancer risk in North Indian population? Urol Oncol 2012.

19. Gordon PM, Soliman MA, Bose P, Trinh Q, Sensen CW, Riabowol K. Interspecies data mining to predict novel ING-protein interactions in human. BMC Genomics 2008, 9:426.

20. Garate $\mathrm{M}$, Wong RP, Campos El, Wang Y, Li G: NAD(P)H quinone oxidoreductase 1 inhibits the proteasomal degradation of the tumour suppressor p33(ING1b). EMBO Rep 2008, 9(6):576-581.

21. Kuo WH, Wang Y, Wong RP, Campos El, Li G: The ING1b tumor suppressor facilitates nucleotide excision repair by promoting chromatin accessibility to XPA. Exp Cell Res 2007, 313(8):1628-1638.

22. Russell MW, Soliman MA, Schriemer D, Riabowol K: ING1 protein targeting to the nucleus by karyopherins is necessary for activation of $\mathrm{p} 21$ Biochem Biophys Res Commun 2008, 374(3):490-495.

23. Garate M, Campos El, Bush JA, Xiao H, Li G: Phosphorylation of the tumor suppressor p33(ING1b) at Ser-126 influences its protein stability and proliferation of melanoma cells. FASEB J 2007, 21(13):3705-3716.

24. Soliman MA, Berardi P, Pastyryeva S, Bonnefin P, Feng X, Colina A, Young D, Riabowol K: ING1a expression increases during replicative senescence and induces a senescent phenotype. Aging Cell 2008, 7(6):783-794

25. Zhu Z, Luo Z, Li Y, Ni C, Li H, Zhu M: Human inhibitor of growth 1 inhibits hepatoma cell growth and influences $p 53$ stability in a variantdependent manner. Hepatology 2009, 49(2):504-512.

26. Han X, Feng X, Rattner JB, Smith H, Bose P, Suzuki K, Soliman MA, Scott MS, Burke $B E$, Riabowol $K$ : Tethering by lamin $A$ stabilizes and targets the ING1 tumour suppressor. Nat Cell Biol 2008, 10(11):1333-1340.

27. Gonzalez L, Freije JM, Cal S, Lopez-Otin C, Serrano M, Palmero I: A functional link between the tumour suppressors ARF and p33ING1. Oncogene 2006, 25(37):5173-5179.

28. Pena PV, Hom RA, Hung $T$, Lin $H$, Kuo AJ, Wong RP, Subach OM, Champagne KS, Zhao R, Verkhusha W, et al: Histone H3K4me3 binding is 
required for the DNA repair and apoptotic activities of ING1 tumor suppressor. J Mol Biol 2008, 380(2):303-312.

29. Vefring HK, Wee L, Jugessur A, Gjessing HK, Nilsen ST, Lie RT: Maternal angiotensinogen (AGT) haplotypes, fetal renin (REN) haplotypes and risk of preeclampsia; estimation of gene-gene interaction from family-triad data. BMC Med Genet 2010, 11:90.

30. Irvin MR, Lynch Al, Kabagambe EK, Tiwari HK, Barzilay Jl, Eckfeldt JH, Boerwinkle E, Davis BR, Ford CE, Arnett DK: Pharmacogenetic association of hypertension candidate genes with fasting glucose in the GenHAT Study. J Hypertens 2010, 28(10):2076-2083.

31. Vangjeli C, Clarke N, Quinn U, Dicker P, Tighe O, Ho C, O'Brien E, Stanton AV: Confirmation that the renin gene distal enhancer polymorphism REN-5312C/T is associated with increased blood pressure. Circ Cardiovasc Genet 2010, 3(1):53-59.

32. Ehret GB, O'Connor AA, Weder A, Cooper RS, Chakravarti A: Follow-up of a major linkage peak on chromosome 1 reveals suggestive QTLs associated with essential hypertension: GenNet study. Eur I Hum Genet 2009, 17(12):1650-1657.

33. Radi ZA, Murad Y: Cellular expression of renal, cardiac and pulmonary inducible nitric oxide synthase in double-transgenic mice expressing human renin and angiotensinogen genes. Clin Exp Pharmacol Physiol 2009, 36(5-6):571-575.

34. Biala A, Tauriainen E, Siltanen A, Shi J, Merasto S, Louhelainen M, Martonen E, Finckenberg P, Muller DN, Mervaala E: Resveratrol induces mitochondrial biogenesis and ameliorates Ang II-induced cardiac remodeling in transgenic rats harboring human renin and angiotensinogen genes. Blood Press 2010, 19(3):196-205.

35. Celoria BM, Genelhu VA, Pimentel Duarte SF, Delfraro PA, Francischetti EA: Hypoadiponectinemia is associated with prehypertension in obese individuals of multiethnic origin. Clin Cardiol 2010, 33(6):E61-65.

36. Paakko T, Ukkola O, Ikaheimo M, Kesaniemi YA: Plasma adiponectin levels are associated with left ventricular hypertrophy in a random sample of middle-aged subjects. Ann Med 2010, 42(2):131-137.

37. Elenkova A, Matrozova J, Zacharieva S, Kirilov G, Kalinov K: Adiponectin - A possible factor in the pathogenesis of carbohydrate metabolism disturbances in patients with pheochromocytoma. Cytokine 2010, 50(3):306-310.

38. Shim CY, Park S, Kim JS, Shin DJ, Ko YG, Kang SM, Choi D, Ha JW, Jang Y, Chung N: Association of plasma retinol-binding protein 4, adiponectin, and high molecular weight adiponectin with insulin resistance in nondiabetic hypertensive patients. Yonsei Med J 2010, 51(3):375-384.

39. Ix JH, Sharma K: Mechanisms linking obesity, chronic kidney disease, and fatty liver disease: the roles of fetuin-A, adiponectin, and AMPK. J Am Soc Nephrol 2010, 21(3):406-412.

40. Persson J, Lindberg K, Gustafsson TP, Eriksson P, Paulsson-Berne G, Lundman P: Low plasma adiponectin concentration is associated with myocardial infarction in young individuals. J Intern Med 2010, 268(2):194-205.

41. Leu HB, Chung CM, Chuang SY, Bai CH, Chen JR, Chen JW, Pan WH: Genetic variants of connexin37 are associated with carotid intimamedial thickness and future onset of ischemic stroke. Atherosclerosis 2011, 214(1):101-106

42. Wilke RA, Simpson RU, Mukesh BN, Bhupathi SV, Dart RA, Ghebranious NR, McCarty CA: Genetic variation in CYP27B1 is associated with congestive heart failure in patients with hypertension. Pharmacogenomics 2009, 10(11):1789-1797.

43. Niu W, Qi Y, Guo S, Gao P, Zhu D: Association of renin Bgll polymphism with essential hypertension: a meta-analysis involving 1811 cases and 1626 controls. Clin Exp Hypertens 2010, 32(7):431-438.

44. Ying CQ, Wang YH, Wu ZL, Fang MW, Wang J, Li YS, Zhang YH, Qiu CC: Association of the renin gene polymorphism, three angiotensinogen gene polymorphisms and the haplotypes with essential hypertension in the Mongolian population. Clin Exp Hypertens 2010, 32(5):293-300.

45. Ragia G, Nikolaidis E, Tavridou A, Arvanitidis KI, Kanoni S, Dedoussis GV, Bougioukas G, Manolopoulos VG: Renin-angiotensin-aldosterone system gene polymorphisms in coronary artery bypass graft surgery patients. $J$ Renin Angiotensin Aldosterone Syst 2010, 11(2):136-145.

46. Ong KL, Li M, Tso AW, Xu A, Cherny SS, Sham PC, Tse HF, Lam TH, Cheung BM, Lam KS: Association of genetic variants in the adiponectin gene with adiponectin level and hypertension in Hong Kong Chinese. Eur J Endocrinol 2010, 163(2):251-257.
47. Kohler S, Bauer S, Horn D, Robinson PN: Walking the interactome for prioritization of candidate disease genes. Am J Hum Genet 2008, 82(4):949-958.

48. Masys DR: Linking microarray data to the literature. Nat Genet 2001, 28(1):9-10.

49. Zhang B, Schmoyer D, Kirov S, Snoddy J: GOTree Machine (GOTM): a webbased platform for interpreting sets of interesting genes using Gene Ontology hierarchies. BMC Bioinformatics 2004, 5:16.

50. WebGestalt (WEB-based GEne SeT AnaLysis Toolkit). [http://bioinfo. vanderbilt.edu/webgestalt/].

51. Aerts S, Lambrechts D, Maity S, Van Loo P, Coessens B, De Smet F, Tranchevent LC, De Moor B, Marynen P, Hassan B, et al: Gene prioritization through genomic data fusion. Nat Biotechnol 2006, 24(5):537-544.

52. Cheng D, Knox C, Young N, Stothard P, Damaraju S, Wishart DS: PolySearch: a web-based text mining system for extracting relationships between human diseases, genes, mutations, drugs and metabolites. Nucleic Acids Res 2008, 36(Web Server issue):W399-405.

53. Zhang B, Kirov S, Snoddy J: WebGestalt: an integrated system for exploring gene sets in various biological contexts. Nucleic Acids Res 2005, 33(Web Server issue):W741-748.

54. Khatri P, Bhavsar P, Bawa G, Draghici S: Onto-Tools: an ensemble of webaccessible, ontology-based tools for the functional design and interpretation of high-throughput gene expression experiments. Nucleic Acids Res 2004, 32(Web Server issue):W449-456.

55. Jensen LJ, Saric J, Bork P: Literature mining for the biologist: from information retrieval to biological discovery. Nat Rev Genet 2006, 7(2):119-129.

56. Chen J, Xu H, Aronow BJ, Jegga AG: Improved human disease candidate gene prioritization using mouse phenotype. BMC Bioinformatics 2007, 8:392.

57. Plake C, Schiemann T, Pankalla M, Hakenberg J, Leser U: AliBaba: PubMed as a graph. Bioinformatics 2006, 22(19):2444-2445.

58. de Bruijn DR, dos Santos NR, Kater-Baats E, Thijssen J, van den Berk L, Stap J, Balemans M, Schepens M, Merkx G, van Kessel AG: The cancerrelated protein SSX2 interacts with the human homologue of a Ras-like GTPase interactor, RAB3IP, and a novel nuclear protein, SSX2IP. Genes Chromosomes Cancer 2002, 34(3):285-298.

59. Turner FS, Clutterbuck DR, Semple CA: POCUS: mining genomic sequence annotation to predict disease genes. Genome Biol 2003, 4(11):R75.

60. Jenssen TK, Laegreid A, Komorowski J, Hovig E: A literature network of human genes for high-throughput analysis of gene expression. Nat Genet 2001, 28(1):21-28.

61. Muller HM, Kenny EE, Sternberg PW: Textpresso: an ontology-based information retrieval and extraction system for biological literature. PLOS Biol 2004, 2(11):e309.

62. Grivell L: Mining the bibliome: searching for a needle in a haystack? New computing tools are needed to effectively scan the growing amount of scientific literature for useful information. EMBO Rep 2002, 3(3):200-203.

63. Tiffin N, Adie E, Turner F, Brunner HG, van Driel MA, Oti M, Lopez-Bigas N, Ouzounis C, Perez-Iratxeta C, Andrade-Navarro MA, et al: Computational disease gene identification: a concert of methods prioritizes type 2 diabetes and obesity candidate genes. Nucleic Acids Res 2006, 34(10):3067-3081.

64. Perez-Iratxeta C, Bork P, Andrade MA: Association of genes to genetically inherited diseases using data mining. Nat Genet 2002, 31(3):316-319.

65. Bada M, Stevens R, Goble C, Gil Y, Ashburner M, Blake JA, Cherry JM, Harris M, Lewis S: A short study on the success of the Gene Ontology. Web Semantics: Science, Services and Agents on the World Wide Web 2004, 1:235-240.

66. Tiffin N, Kelso JF, Powell AR, Pan H, Bajic VB, Hide WA: Integration of textand data-mining using ontologies successfully selects disease gene candidates. Nucleic Acids Res 2005, 33(5):1544-1552.

67. Wren JD, Garner HR: Shared relationship analysis: ranking set cohesion and commonalities within a literature-derived relationship network. Bioinformatics 2004, 20(2):191-198.

68. Entrez Gene FTP. [ftp://ftp.ncbi.nih.gov/gene/DATA]

69. Becker KG, Barnes KC, Bright TJ, Wang SA: The genetic association database. Nat Genet 2004, 36(5):431-432.

70. Gene Ontology OBO data. [http://geneontology.org/ontology/ obo_format_1_2/.

71. GNU GPL. [http://www.gnu.org/licenses/\#GPL]. 
doi:10.1186/1471-2164-13-S8-S20

Cite this article as: Jourquin et al:: GLAD4U: deriving and prioritizing

gene lists from PubMed literature. BMC Genomics 2012 13(Suppl 8):S20.

Submit your next manuscript to BioMed Central and take full advantage of:

- Convenient online submission

- Thorough peer review

- No space constraints or color figure charges

- Immediate publication on acceptance

- Inclusion in PubMed, CAS, Scopus and Google Scholar

- Research which is freely available for redistribution

Submit your manuscript at 